\title{
ReFrAEN: a Reconfigurable Vibration Analysis Framework for Constrained Sensor Nodes
}

\author{
Ashok Samraj Thangarajan \\ Fan Yang \\ Wouter Joosen \\ Sam Michiels \\ imec-DistriNet, KU Leuven \\ imec-DistriNet, KU Leuven imec-DistriNet, KU Leuven imec-DistriNet, KU Leuven \\ ashoksamraj.thangarajan@kuleuven.be fan.yang@kuleuven.be \\ wouter.joosen@kuleuven.be sam.michiels@kuleuven.be
}

\author{
Danny Hughes \\ imec-DistriNet, $K U$ Leuven \\ danny.hughes@kuleuven.be
}

\begin{abstract}
Vibration monitoring uses data gathered from accelerometers to study kinetic phenomena in applications such as: structural health monitoring and predictive maintenance. The Internet of Things (IoT) has the potential to greatly expand the range and scope of vibration monitoring applications by delivering long-life wireless sensors that can be cost-effectively embedded in hard to reach places such as; within machines, infrastructure or the built environment. However, achieving this vision is difficult due to the stringent resource constraints of contemporary IoT devices and networks. This has led the research community to develop a creative range of applicationspecific near-sensor processing firmware. However, systematic support for generic vibration monitoring on resource-poor IoT networks remains an open problem. We tackle this challenge by introducing $\operatorname{ReFrAEN}$, a software framework that efficiently enables a wide range of vibration monitoring applications on IoT networks. ReFrAEN achieves this through a deeply configurable combination of compression techniques and data processing algorithms. These features allow end-users to effectively tradeoff between resource consumption and data resolution in order to meet battery life constraints while preserving sufficient data quality to support the target application. Our evaluation shows that $\operatorname{ReFrAEN}$ is capable of identifying bearing faults, while dramatically improving battery lifetime and reducing latency in comparison to prior approaches.
\end{abstract}

Index Terms-IoT, Near Sensor Processing, Vibration Monitoring

\section{INTRODUCTION}

Vibration monitoring is used in a wide range of applications, such as Structural Health Monitoring (SHM) of wind turbines and bridges [1], [2] and Condition Based Maintenance (CBM) of industrial machines [3]. Many of these application scenarios require data to be collected from remote locations, bringing new challenges to system design and deployment, such as: (i.) system installation where power or networking is unavailable, (ii.) high maintenance cost and complexity, and (iii.) enabling deployment within dense machinery or on rotating equipment where cabling is infeasible.

Wireless Internet of Things (IoT) technologies have become a key enabler in gathering industrial telemetry [4], [5]. State of the art IoT devices can perform simple sensing operations on a single battery charge for several years [6]. However, IoT networks trade-off bandwidth to achieve a better energy profile [7]. As a result, both long range (e.g. LoRa, and SigFox
[7]-[9]) and short range networks (e.g. 6TiSCH and Bluetooth Mesh [10], [11]) have strict bandwidth limitations. Moreover, it is not possible to increase the performance of these networks without reducing battery life. For example, Sabato et al. [1] show that the key impediment to long term wireless vibration monitoring is battery life, and that up to $75 \%$ of battery consumption is due to the networking. Furthermore, transmitting large amounts of data causes network congestion and packet loss. To mitigate the contradiction between energy and bandwidth flexible data analysis must be performed on the embedded end-device itself. However, identifying faults locally on-node [12], [13] has problems such as highly customized solutions for the application, lack of historical maintenance data and distinguishing failure of deployed sensors and machines faults [14], [15].

In this paper, we propose ReFrAEN, a generic vibration monitoring framework that delivers deep reconfiguration support of sensor parameters, a suite of analysis algorithms and compression techniques. These techniques significantly reduce bandwidth requirements and enable the selective extraction of high resolution spectra to support narrow band analysis and variable band harmonic analysis [16]. Furthermore, the computationally light compression technique improves the signal to noise ratio, similar to tradition approaches such as noise cancellation and adaptive line enhancer [17], which are not feasible on a constraint end-node. The contributions of ReFrAEN are three-fold: (i.) A reconfigurable vibration monitoring framework for embedded IoT devices. (ii.) Novel on-node processing algorithms to scale and compress data. (iii.) Programmable frequency band extraction for targeted analysis.

We evaluate ReFrAEN on an open set of representative vibration data and demonstrate its capability to reduce data transmissions by over $68 \%$ while still identifying faults in real time. Critically, ReFrAEN improves the lifetime of IoT devices by over $75 \%$ in comparison to current methods while reducing latency by over $66 \%$ in comparison to the transmission of standard spectrum data.

The remainder of the paper is organized as follows. In Section II, we provide background on vibration monitoring using wireless sensor networks and derive the requirements for 
ReFrAEN. Section III then describes the design of ReFrAEN and in Section IV a reference implementation is provided. In Section V, we evaluate the performance of ReFrAEN. In Section VI, we discuss related work and highlight the gap that ReFrAEN addresses. Finally, Section VII provides concluding remarks and future work.

\section{IOT VIBRATION MONITORING}

Vibration monitoring is widely used in application domains such as Condition Based Maintenance (CBM) and Structural Health Monitoring (SHM). Vibration monitoring is a particularly effective technique to identify faults in industrial machines as faults tend to manifest earlier than in temperature or electrical current signals. Using wireless IoT devices dramatically lowers deployment costs and opens up the possibility of instrumenting existing infrastructure with minimal disturbance to system operation [18]-[21]. This section provides background on the network, computation and software requirements of IoT vibration monitoring. Section II-D then distills requirements for ReFrAEN.

\section{A. Network Requirements}

Vibration monitoring generates large amounts of data in comparison to the limited bandwidth of IoT networks. For example, a typical accelerometer sensor that is used for vibration monitoring may sample at $1-20 \mathrm{KHz}$ in a resolution of $2 \mathrm{~B}$, generating $2-40 \mathrm{kBps}$. This is an enormous load for conventional IoT networks. For example, consider the Low Power Wide Area Network (LPWAN) technologies LoRa and Sigfox. In its longest range configuration, LoRa offers a maximum bandwidth of 290bps, which is further reduced by a factor of 10-1000x depending upon regional duty cycle limitations [22]. This results in an effective bandwidth of between 0.29 to $29 \mathrm{bps}$. Similarly, Sigfox connections are limited to 140 upstream packets per day with a maximum application payload of 12 bytes [7], resulting in an average bandwidth of $0.019 \mathrm{bps}$. While the performance of multihop mesh networks is better, they still fall far short of the bandwidth that is required to transmit raw accelerometer telemetry [12], [23]. Furthermore, sending large blocks of data over IoT networks causes contention which leads to either packet loss and increases bandwidth requirements to support reliable/acknowledged transmission.

It should be noted that IoT network performance cannot be further improved without negatively impacting battery life, and thereby increasing maintenance costs due to battery replacement [24], [25]. On-device vibration analysis is thus required to transform raw data into finished analyses and thereby reduce bandwidth requirements.

\section{B. Processing Requirements}

IoT end nodes have a limited energy budget that is constrained by their battery size or energy harvesting capability [24], [26]. This limits the computational and memory resources of the devices and forms a barrier to the use of the complex algorithms that are required for vibration analysis. Common mote platforms such as MicroPnP, Telos and
Permamote [27]-[29] offer processor speeds that range from a few Million Instructions Per Second (MIPS) to few hundred MIPS. This is several orders of magnitude less compute power than is available on a typical personal computing device. Nevertheless, the power of IoT devices is increasing rapidly.

The original TMote from UC Berkeley had $10 \mathrm{kB}$ RAM and ran at 8 MIPS [28]. In contrast, the more recent Nordic nRF52832 [30] processor that is used for our prototype, embeds a Cortex-M4F processor with $64 \mathrm{kB}$ of RAM running at 80 MIPS. A 1024-point Fast Fourier Transform (FFT) takes approximately $2.2 \mathrm{~ms}$ and $2 \mathrm{kB}$ of memory (assuming $2 \mathrm{~B}$ per sample), consuming $26 \mu \mathrm{J}$ of energy. While it is clear that the limited energy and performance of IoT end devices requires careful engineering, this is several orders of magnitude less energy than is required to send $2 \mathrm{kB}$ of data over the radio $(2.22 \mathrm{~mJ})$, making a strong case for IoT near-sensor analysis.

\section{Near-sensor Analysis Software}

Vibration monitoring involves various tools for time and frequency domain analysis which enable engineers to distinguish between healthy and faulty machines. Statistical data analyses such as Root Mean Square (RMS), crest factor and kurtosis generates smaller amounts of data, however, they have limited success in detecting subtle faults [31]. On the other hand, spectrum analysis techniques such as Fast Fourier Transform (FFT) have a proven track record of supporting diverse CBM applications [31], [32]. Given the stringent bandwidth requirements of IoT systems, these solutions must also be deeply parameterizable in order to enable application developers to focus their limited network resources on examining the specific phenomena of interest.

It is common practice to use vibration monitoring to identify faults at known frequencies. For example in the case of bearing analysis, it is common to monitor the frequencies of inner race, outer race and roller faults, all of which can be calculated using the bearing specifications and shaft speed [33]. While these frequencies may be selectively extracted in order to reduce the overall bandwidth requirements of the application, the frequencies of interest vary along with shaft speed over time. This demands reconfiguration support in order to focus analysis upon the changing phenomena of interest. These results may then be used as input to standard analyses in the back end such as narrow band analysis, variable band harmonic analysis and band frequency measurement [16].

\section{Requirements}

Based upon the analysis presented above, we identify the following key requirements for ReFrAEN:

1) To minimize network load, finished analyses must be sent rather than raw data [7], [22].

2) Compression and filtering algorithms are required to maximize the efficiency with which analyses are presented [1].

3) It is necessary to extract select spectrum bands in configurable resolution to support common CBM analyses [16]. 


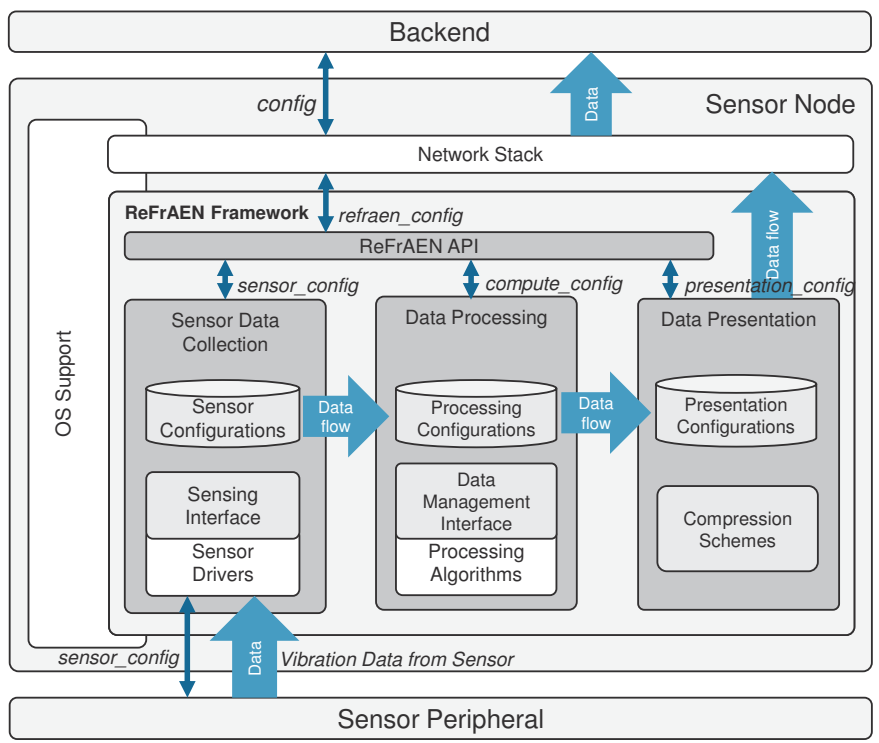

Fig. 1: Block diagram of ReFrAEN with high level interaction

4) The framework must operate within the resource constraints of contemporary IoT processors in terms of computation complexity, RAM and ROM.

The following section introduces the design of ReFrAEN, an embedded vibration analysis toolkit that addresses these requirements.

\section{DESIGN}

The design of ReFrAEN enables selective data extraction for fault identification over low bandwidth IoT networks by optimizing data collection and presenting only essential information. The block diagram and high level interactions of ReFrAEN are shown in Figure 1. Sensor data collection is optimized to the specifications of the application (e.g. to cover known fault frequencies) and data transformation is optimized in synergy with data collection to deliver high resolution data without compromising efficiency. ReFrAEN can be configured to provide a summary of the system under test in single packet that we call summary spectrum or high resolution details through selective spectrum extraction. In the next sections, we will describe the front-end optimizations on sensor data collection and processing, followed by the modes of data presentation using summary and selective spectrum extraction methods. We then provide the remote APIs of ReFrAEN that enable back-end interaction with the sensor node.

\section{A. Sensor Reconfiguration}

ReFrAEN is designed for use with digital accelerometers. These sensors support a wide range of reconfiguration parameters, which allow their behavior to be tailored to suit the requirements of specific CBM or SHM applications:

1) Axis: many accelerometers support sensing in up to three dimensions. ReFrAEN allows the developer to select specific a specific axis of interest and additionally supports averaged analyses combining all axes and roundrobin analysis on each axis in turn.
2) Measurement range is often configurable, enabling high resolution sensing of small amplitude acceleration data or low resolution sensing of large amplitude acceleration data. ReFrAEN exposes these parameters.

3) Sample rate may also be configurable. ReFrAEN will automatically select the most appropriate sample rate based upon the developer's specifications.

4) Resolution may be configurable from $8 b$ to $32 \mathrm{~b}$ depending upon the capabilities of the sensor. ReFrAEN will automatically select the optimum resolution based upon developer requirements.

The need for configurability arises due to IoT resource limitations and divergent application requirements. Considering resource limitations: higher resolutions generate more data and use more energy. Considering application requirements: some phenomena may only be visible in a single axis or measurement range. The state of practice in near-sensor vibration analysis is to hard-code an application-specific configuration that conserves resources while capturing the phenomena of interest. ReFrAEN takes an alternative approach, supporting runtime reconfiguration of the accelerometer. This enables a sensor to be dynamically re-tasked as requirements change.

\section{B. Data Processing}

ReFrAEN provides a library of data processing algorithms in both the time and frequency domains:

1) Root-Mean-Square (RMS): is a well known time-domain analysis that reveals the energy content of a vibration signal.

2) Impact Detection is a time-domain analysis that records the largest single acceleration event within a given timeperiod, recording its magnitude in multiple axis in order to identify its potential origin.

3) Fast Fourier Transform (FFT) provides an efficient method to transform time-domain data to frequency domain data to support various analyses. The ReFrAEN FFT library offers configurable resolution from 32 to 4096 bins with 4 to 32 bit bin width. While higher resolution may reveal more detail, they also increase data storage and transfer requirements.

The development of additional data processing algorithms is the subject of our future work, as explored in Section VII. As with the accelerometer sensor, which specific analyses to apply and in what configuration may be reconfigured at runtime in order to adapt to changing requirements or support multiple applications simultaneously.

\section{Data Compression and Presentation}

ReFrAEN provides a range of data compression and presentation techniques, which condition the output of the data processing tools described in Section III-B to fit within the available packet size of the host IoT network.

1) Amplitude Compression: Algorithm 1 shows the method used to scale and compress the spectrum. Amplitude scaling works by finding the maximum frequency bin amplitude and generating a scaling factor that matches this value to the 


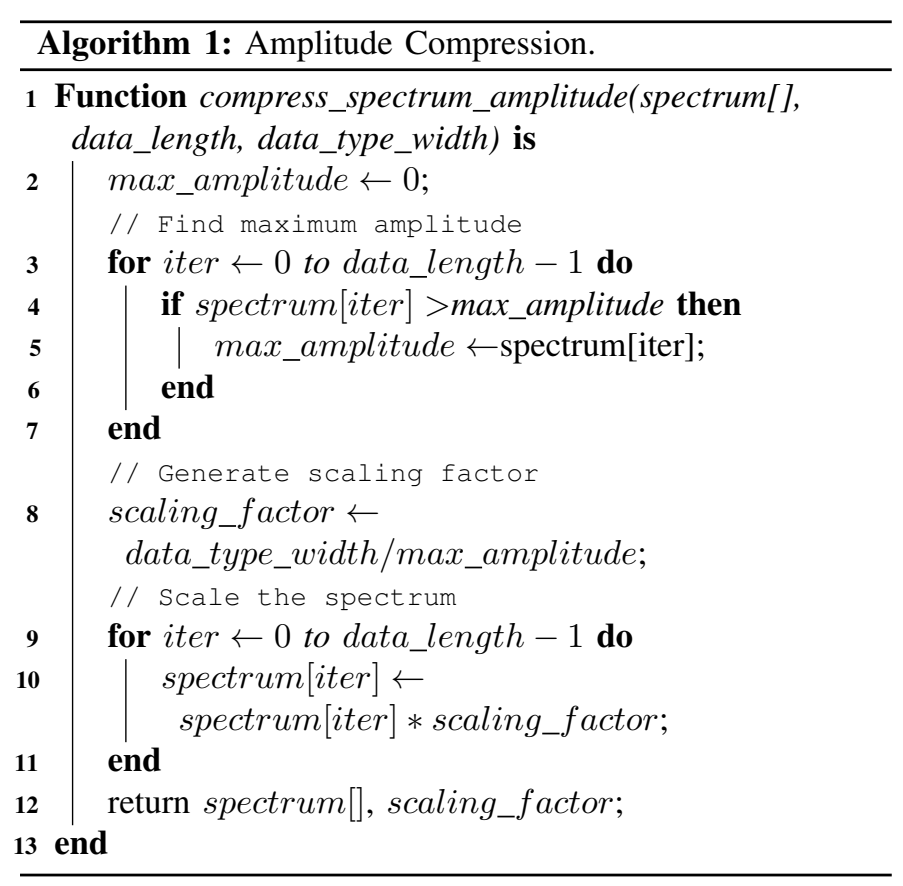

output datatype width. The spectrum is then scaled to fit the datatype, which can be restored by doing the inverse operation on the spectrum, with the scaling factor that is transmitted along with the spectrum. For instance, in the representative accelerometer [34] used in our evaluation, each data point has a resolution of two bytes, which ReFrAEN can further downscale to 4 or 8 bits per bin. The degree of compression can be selected during at run time using the ReFrAEN APIs.

2) Selective Spectrum Extraction: When the backend requires high resolution data from a specific band, the ReFrAEN stack is reconfigured to filter the FFT output. The bands start and end frequencies are given and a high resolution spectrum of the band is then extracted using Algorithm 2. The selective spectrum extraction methods works like a band-pass filter in frequency domain.

3) Frequency Compression: When the requested spectrum data cannot be accommodated by the maximum packet size of the IoT network, the spectrum is further compressed in the frequency domain to produce a summary of the requested spectral data. The Algorithm 3 provides the method to generate the summary spectrum. The generated summary spectrum gives an overview of system behaviour at a reduced bin resolution. If $F_{S}$ is the sampling frequency, NFFT is the FFT size, $L_{S}$ represents the length of the spectrum in bytes and $L_{P}$ represents packet length in bytes. The reduced bin resolution $B_{\text {RES }}$ is then given by:

$$
B_{R E S}=\left(F_{s} / N F F T\right) *\left(L_{\mathrm{S}} / L_{\mathrm{P}}\right)
$$

\section{ReFrAEN API}

The remote APIs provided by ReFrAEN are categorised into sensor, compute and data presentation. Table I lists the APIs in each categories. Each set method has a corresponding get method, which returns the current settings. This is omitted in the interests of brevity. In addition to the manual configuration options, the stack provides a self configuration mode
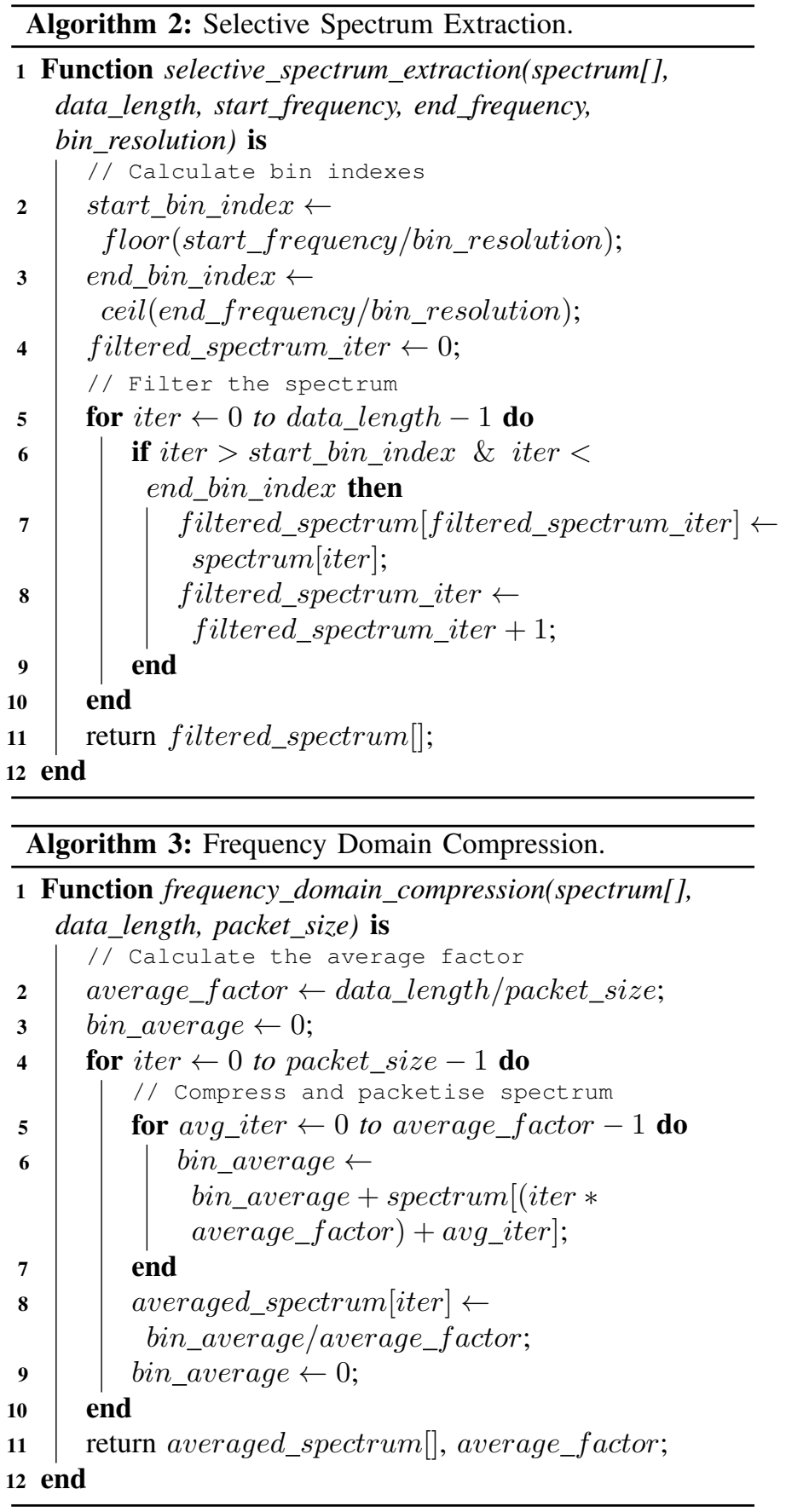

in which, the maximum frequency that should be monitored (max_frequency) and the required frequency resolution is provided. The sensing and computation parameters are then self-configured to maximize efficiency. For instance, if the selected max_frequency is $200 \mathrm{~Hz}$ and the required resolution is $1 \mathrm{~Hz}$, the minimum compatible sampling rate available on the LSM6DS3 [34] that satisfies Nyquist criteria is $416 \mathrm{~Hz}$ and a 512-point FFT will be selected which delivers a frequency resolution of $0.81 \mathrm{~Hz}$

\section{IMPLEMENTATION}

The ReFrAEN stack was implemented on two representative MCUs, the nRF52832 [30] and ATMega1284p, the 
TABLE I: APIs for Configuring ReFrAEN Stack

\begin{tabular}{lll}
\hline ReFrAEN Block & API & Parameter Values \\
\hline \multirow{4}{*}{ Sensor Configs } & set_axis & X, Y, Z, All, Rotate \\
& set_range & $2 \mathrm{~g}-16 \mathrm{~g}$ \\
\cline { 2 - 3 } & set_sampling_rate & $52 \mathrm{~Hz}-6.67 \mathrm{KHz}$ \\
& set_sensor_resolution & $8-32$ bits \\
\hline \multirow{4}{*}{ Compute Configs } & set_rms & enable/disable \\
& set_impact_detection & enable/disable \\
\cline { 2 - 3 } & set_fft_size & $32-4096$ \\
& set_fft_bin_resolution & $4-32$ bits \\
\hline \multirow{2}{*}{ Presentation Configs } & selective_frequency_extraction & start_frequency, end_frequency \\
& refraen_self_configure & max_frequency, bin_resolution \\
\hline
\end{tabular}

TABLE II: Configuration of the Microcontrollers in which ReFrAEN was Implemented

\begin{tabular}{|l|c|c|c|c|c|}
\hline & $\begin{array}{c}\text { Clock Speed } \\
(\mathrm{MHz})\end{array}$ & $\begin{array}{c}\text { Flash } \\
(\mathrm{KB})\end{array}$ & $\begin{array}{c}\text { RAM } \\
(\mathrm{KB})\end{array}$ & $\begin{array}{c}\text { Active Current } \\
(\mathrm{mA})\end{array}$ & $\begin{array}{c}\text { Sleep Current } \\
(\mathrm{uA})\end{array}$ \\
\hline nRF52832 & 64 & 512 & 64 & 3.7 & 0.3 \\
\hline ATMega1284p & 4 & 128 & 16 & 2.5 & 0.4 \\
\hline
\end{tabular}

specifications of which are listed in Table II. ReFrAEN is independent of wireless technology, and the only requirement on the chosen network is support for downlink messages. The accelerometer sensor included for the power measurements is LSM6DS3 from STMicroElectronics [34], and the representative reconfiguration parameters provided by the sensing interface are implemented to match the device specifications. The accelerometer is powered on and configured at the start of each sensing cycle. New configuration received in-between an ongoing cycle is applied in the next sense cycle, avoiding any negative impacts of reconfiguration. The energy estimates for LPWAN networks were taken from prior studies of LoRa [35], NB-IoT [7] and Sigfox [36]. The node is powered by a 3.6V, $2700 \mathrm{mAh}$ battery [37]. We implemented a custom back-end to perform fault diagnosis on collected data.

\section{Evaluation}

In this section we evaluate the ability of ReFrAEN to accurately and efficiently identify faults in vibration data using three representative IoT networks (LoRa, SigFox and NBIoT). The vibration data used in our experiments is drawn from the Case Western Reserve University (CWRU) bearing data set [38], a widely used reference in the field [39]. The dataset was collected at a sample rate of $12 \mathrm{KHz}$ under three fault conditions (inner race, outer race and ball fault) and at four rotation speeds (1730, 1750, 1772 and 1797 RPM). The fault frequencies at different shaft speeds are obtained from the fault specification provided in the CWRU repository [38]. The state of practice in fault diagnosis using vibration monitoring requires a frequency resolution of at least $1 \mathrm{~Hz}$ [16]. We assume a sensor network that has a packet size of 32 bytes for all experiments.

\section{A. Bearing Vibration Analysis}

This section demonstrates how ReFrAEN is used on the CWRU vibration dataset to extract the features that are required to monitor bearing fault frequencies. The analysed vibration data-set is approximately 10 seconds in length, at a shaft speed of 1797 RPM with an inner race defect of 0.021 inches. Additional data-sets collected at 1730, 1750 and 1772 RPM with the same fault conditions are used as required. We identify faults using a peak ratio threshold [17], [40], wherein the peak ratio is calculated by dividing the peak amplitude of the fault frequency by the average of the spectrum. We set a fault detection threshold at twice the non-faulty peak ratio, which is sufficient to identify all faults in the data set.

Accelerometer Reconfiguration: Sensor reconfiguration is simulated by downsampling the data to one of the sample rates available on the LSM6DS3. The fault frequencies as given by the CWRU data bearing specifications [38] all fall below $200 \mathrm{~Hz}$. Accordingly, the sensor is reconfigured to sample at $416 \mathrm{~Hz}$ and a 512-point FFT is computed, which gives a maximum frequency resolution of $0.81 \mathrm{~Hz}$. Initially, no specific frequency bands were configured for extraction and ReFrAEN thus provides a summary spectrum with $8 \mathrm{~b}$ amplitude resolution that is compressed in frequency domain, resulting in a bin width of $6.5 \mathrm{~Hz}$ (Section III-C3) as shown in Figure 2.

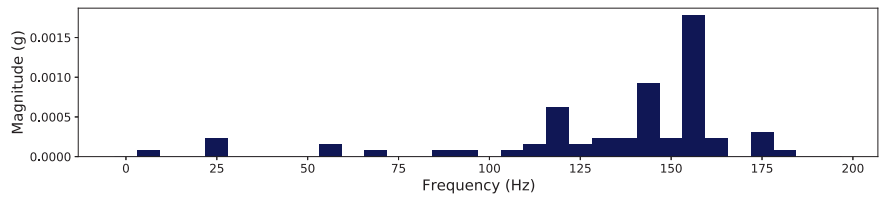

Fig. 2: Summary Spectrum for Dataset with IR Fault and Shaft Speed of $1797 \mathrm{rpm}$.

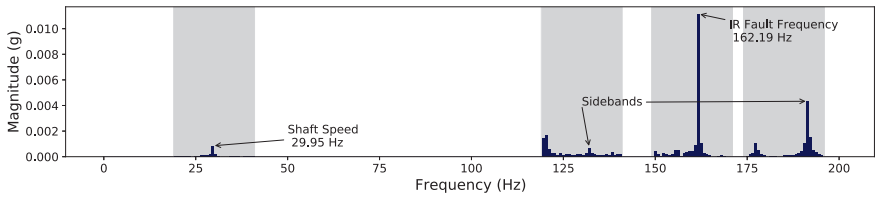

Fig. 3: Ensemble of Sample Periods Showing Four Extracted Fault Features.

Fault Frequency Extraction: The inner race fault frequency is at $162.19 \mathrm{~Hz}$, with side-bands at $132.24 \mathrm{~Hz}$ and 192.14 Hz. The motor rotation signal is also available in the FFT at $29.95 \mathrm{~Hz}$. ReFrAEN is therefore configured to extract four $20 \mathrm{~Hz}$ sub-spectra $(20-40 \mathrm{~Hz}, 120-140 \mathrm{~Hz}, 150-170 \mathrm{~Hz}$ and $175-195 \mathrm{~Hz})$ that, in ensemble, describe all relevant data. Each sub-spectrum is encoded with $8 \mathrm{~b}$ amplitude resolution, requiring 27 bytes ( 25 bytes for the spectrum data and 2 bytes for the scaling factor). This is within the available 32B packet size. The resulting four packet ensemble spectrum is shown in Figure 3. As can be seen from the figure, selective frequency extraction significantly improves the readability of data.

Fault Identification: As described previously, faults were identified using a peak ratio threshold. The peak ratio is first calculated by dividing the peak amplitude of the fault frequency by the average of the spectrum [17], [40], where the spectrum average is calculated from the low resolution spectrum at 8 bit bin width as shown in Figure 2, and the defect peak is extracted from the ensemble band containing $162.19 \mathrm{~Hz}$. A threshold of twice the non-faulty peak ratio was used to identify faults. To validate the consistency of this method, the peak ratio of faulty data-sets was analysed for shaft speeds of 1730, 1750 and 1772 RPM. The threshold was sufficient to identify faults in all cases. 

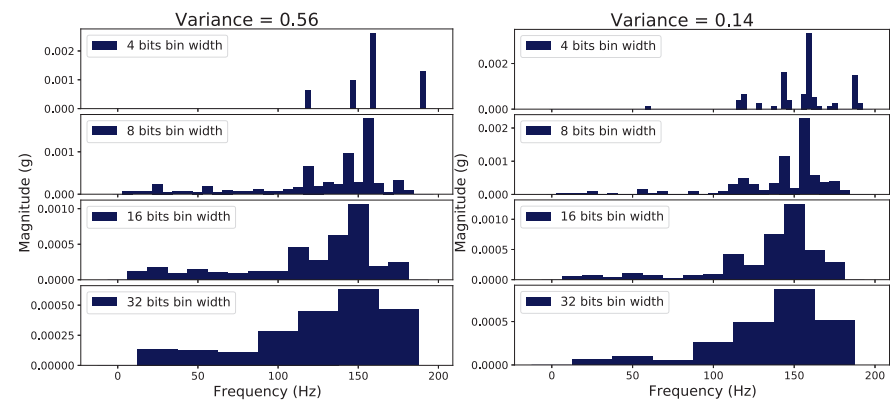

Fig. 4: Frequency Compressed Spectrum with Different Bin Widths
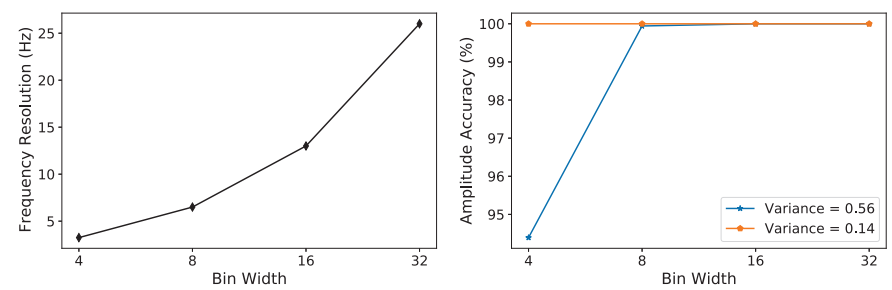

Fig. 5: Frequency (left) and Amplitude (right) Resolution Vs Bin Width.

\section{B. Accuracy}

In this section, we first visually explore the impact of amplitude and frequency compression on accuracy. Following this, we assess the degree to which selectively extracted spectra correlate with the uncompressed and high resolution spectrum, hereafter referred to as the raw spectrum.

Trading-off Amplitude and Frequency Resolution: Figure 4 shows a single packet (i.e. 32B) summary spectrum that was created with bin resolutions of 4 to $32 \mathrm{~b}$, resulting in a bin width of 3.25 to $26 \mathrm{~Hz}$. The output is visualised for shaft speeds of 29.95 and $29.53 \mathrm{~Hz}$, which has a data variance of 0.56 and 0.14 respectively (we refer the reader to [41] for the calculation of data variance). It can be seen that a smaller bin width results in higher accuracy in the frequency domain at the cost of accuracy in the amplitude domain. Figure 5 (left) shows the drop in frequency resolution that occurs as bin width increases. Figure 5 (right) compares amplitude accuracy at the fault frequency, for amplitude compressed spectra that is scaled to different bin widths, at a frequency resolution of $0.81 \mathrm{~Hz}$. As can be seen from the figure, the scaling techniques of ReFrAEN perform better on data with a lower variance. For the data-set with a variance of 0.56 , it can be seen that an $8 \mathrm{~b}$ bin width provides $99.94 \%$ accuracy at the fault frequency for a frequency resolution of $6.5 \mathrm{~Hz}$ in the summary spectrum. The accuracy drops to $94.39 \%$ at $4 \mathrm{~b}$ bin width while providing a higher frequency resolution of $3.25 \mathrm{~Hz}$. However, the spectrum is sparser as small magnitude signals are hidden due to the reduced resolution. By offering configurable amplitude scaling, ReFrAEN enables the developer to intelligently tradeoff accuracy between the frequency and amplitude domains.

Accuracy of Selectively Extracted Spectra: Figure 3 shows the ensemble sum of the four extracted sub-bands representing the fault frequency, side-bands and the shaft rotation speed. To assess the accuracy of our approach, we extracted similar subbands from the high resolution spectrum $(0.81 \mathrm{~Hz})$ obtained
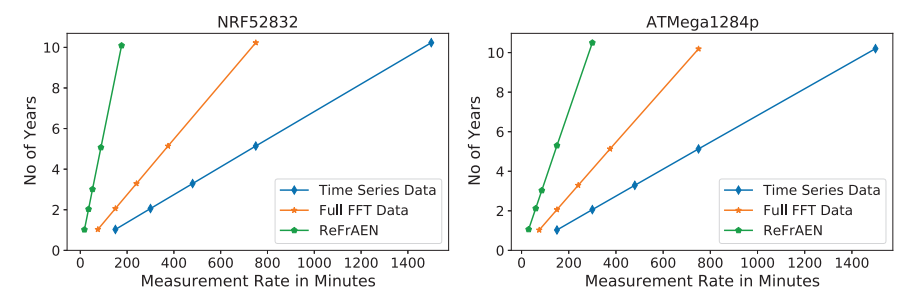

Fig. 6: Sensor Node Lifetime for nRF52832 and ATMega1284p Deployed with 2700mAh Battery.

using the standard Python FFT library running on a laptop and compared the results using correlation analysis. Our results show that the two methods are $97.89 \%$ correlated in frequency band $20-40 \mathrm{~Hz}, 99.53 \%$ in $120-140 \mathrm{~Hz}$ band, $99.98 \%$ in $150-170 \mathrm{~Hz}$ band and $99.78 \%$ in $175-195 \mathrm{~Hz}$ band. The high correlation shows that the loss in accuracy due to the compression techniques that are used to extract signal is sufficiently low that it should not be expected to effect the fault diagnosis process.

\section{Network Efficiency}

To evaluate network efficiency, we compare the impact of using ReFrAEN to send only those features that are required to diagnose faults against the transmission of raw time series or spectrum data using three representative IoT networks: LoRa, SigFox and NB-IoT.

In all cases, each data point is measured at $2 \mathrm{~B}$ resolution and at a sample rate of $416 \mathrm{~Hz}$. A 512 bin FFT is then performed on the data. Sending time-series data requires the transmission of $1 \mathrm{kB}$ of raw samples. Transmitting complete spectrum data requires 512B of FFT data. In the case of ReFrAEN, transmitting four fault-related artefacts (corresponding to four possible faults in bearings [38]) require the transmission of $160 \mathrm{~B}$ of data; 32B to send the summary spectrum and 32B each to send the four fault sub-spectra. As described in Section V-A, this is sufficient for accurate fault diagnosis across all case-study data. This is an improvement of $84.38 \%$ over transmitting raw time-series data and $68.75 \%$ over spectrum data.

\section{Energy Efficiency}

We compare the battery life impact of ReFrAEN against the transmission of raw time-series and spectrum data for the nRF52832 and ATMega1284p, deployed with a 2700mAh battery and LoRa radio. The LoRa network was configured in its longest range setting at Spreading Factor (SF) 12, with a $1 \%$ duty cycle as described in [35]. Figure 6 shows the rate at which measurements that can be taken in order to achieve a given network lifetime using ReFrAEN vs raw time-series or spectrum data. For a 10 year lifetime, one measurement can be taken approximately every 176.5 minutes using the nRF52832 and every 300 minutes using the ATMega1284p using ReFrAEN.

In order for appropriate intervention to be scheduled, faults must be identified at stage 3 as defined in [41], [42]. Stage 3 affords 30-40 days of intervention time, while stage 4 is infeasible as it requires immediate intervention. We compare the time required to extract diagnosable data using ReFrAEN 
TABLE III: Time Taken to Transfer Diagnosable Data

\begin{tabular}{|c|c|c|c|c|c|c|c|c|c|}
\hline & $1 \mathrm{Yr}$ & $5 \mathrm{Yrs}$ & $10 \mathrm{Yrs}$ & $1 \mathrm{Yr}$ & 5 Yrs & 10 Yrs & 1 Yr & 5 Yrs & 10 Yrs \\
\hline $\begin{array}{c}\text { Time Series } \\
\text { (in hours) }\end{array}$ & 2.5 & 12.5 & 25 & 2.5 & 33.3 & 66.7 & 25 & 123.1 & 246.2 \\
\hline $\begin{array}{c}\text { Full Spectrum } \\
\text { (in hours) }\end{array}$ & 1.25 & 6.25 & 12.5 & 1.25 & 16.7 & 33.3 & 12.5 & 61.5 & 123.1 \\
\hline $\begin{array}{c}\text { ReFrAEN } \\
\text { (in hours) }\end{array}$ & 0.29 & 1.47 & 2.94 & 1.05 & 5.26 & 10.53 & 4.17 & 20.83 & 41.67 \\
\hline
\end{tabular}

TABLE IV: ReFrAEN Performance Timings

\begin{tabular}{|c|c|c|c|}
\hline & $\begin{array}{c}\text { Amplitude } \\
\text { Compression (ms) }\end{array}$ & $\begin{array}{c}\text { Frequency Domain } \\
\text { Compression (ms) }\end{array}$ & $\begin{array}{c}\text { Selective Spectrum } \\
\text { Extraction (ms) }\end{array}$ \\
\hline nRF52832 & 0.118 & 0.0608 & 0.0478 \\
\hline ATMega1284p & 21.85 & 114 & 68.32 \\
\hline
\end{tabular}

vs raw time-series and spectrum data over three LPWAN networks LoRa, Sigfox and NB-IoT. Network performance and configuration was taken from the literature [7], [22], [35], [36]. The amount of data that each network can transmit is constrained by the available power budget and the required device lifetime. From Table III it can be seen that ReFrAEN can deliver diagnostic data up to $88.24 \%$ faster than traditional approaches to guarantee scheduling of intervention at stage 3 of bearing failure for all LPWAN networks and for required lifetimes of up to 10 years.

\section{E. Performance Timings}

Table IV lists the performance timings of ReFrAEN for two microcontrollers. As in the bearing fault diagnosis example, the framework was configured to perform a 512-point FFT that generates 256 bins, with amplitude compression generating an $8 \mathrm{~b}$ bin width. Frequency compression packs the data to a buffer of size 32 bytes for transmission. A $20 \mathrm{~Hz}$ sub-band was then extracted using selective spectrum extraction. The overhead of frequency domain compression is proportional to the packet size, selective spectrum extraction to bandwidth and amplitude compression to the length of data and bin width.

\section{RELATED WORK}

This section reviews the state of the art in wireless vibration monitoring for Condition Based Maintenance (CBM) and Structural Health Monitoring (SHM).

Jung et al. [23] proposed a data analysis approach to estimate Remaining Useful Life (RUL). Their wireless IoT device records 1024 data points along three axis in one sampling period and partitions the data into 120 packets to transfer to the back-end using the reliable Flush protocol [43]. This method is reliable, but requires acknowledgments for each packet, which increases energy consumption and is not supported by some LPWAN networks [7]. Moreover, as we have shown, transferring raw time series data using IoT networks is inefficient. Unlike ReFrAEN, The authors do not employ data analysis techniques on the end-nodes. Similarly, most sensor network deployments in CBM [44]-[46] and SHM [1], [21] applications use IoT devices only for data acquisition and collection and do not leverage the increasingly capable computing power of IoT devices.

Several researchers have investigated the need for nodelocal processing to extract features and to conserve energy [47]-[49], however, they typically conclude that FieldProgrammable Gate Arrays (FPGA) and custom Systemon-Chips (SoC) are required to enable adequate node-local processing and reconfiguration. ReFrAEN shows that this is possible in software by optimizing node-local processing together with deep configurability of the sensing and computing firmware of the IoT device to selectively present essential data.

An orthogonal stream of research performs feature extraction on the IoT device itself [18], [19], [24], or adopt the approach of diagnosing and identifying faults locally on the sensor node [12], [13]. This improves energy consumption and node lifetime by reducing the amount of data to be transmitted. However, such sensor nodes have the following problems (i.) the data resolution that they obtain does not support classic fault identification algorithms [41], [42], [50], (ii.) the solution is tailored for a specific application and is not possible to re-task the node to perform other applications over their long lifetime [26], [51], (iii.) large-scale deployments require a history of the machine health, which is not facilitated by pure on-device fault identification. ReFrAEN solves the problem with a run-time reconfigurable framework that can interactively extract features for fault diagnosis in a variety of applications.

\section{CONCLUSION}

This paper proposes the ReFrAEN framework for embedded vibration analysis. ReFrAEN enables deep reconfigurability of sensing and the computation components, while providing a suite of data analysis and compression algorithms through which high resolution vibration data can be extracted over low power, low bandwidth IoT networks. Evaluation of ReFrAEN on a standard open data set combined with implementations for two representative IoT platforms illustrate that our approach dramatically reduces bandwidth and energy requirements as well as the latency with which diagnosable data can be extracted. This is accomplished while preserving sufficient data quality to support a real-world Condition Based Monitoring (CBM) case-study.

In its current form, ReFrAEN requires the user to know the frequency bands of interest to provide high resolution data. As future work, we will explore methods to automatically identify spectrum bands of interest from the low resolution spectrum data and then automatically extract high resolution data for diagnosis, using an optimal number of transmissions. ReFrAEN is limited to extracting data over a long period of time and hence is suitable for faults that has long intervention periods. Sporadic and ephemeral faulty events cannot be monitored using methods provided by ReFrAEN. To address this shortcoming, we will explore methods to monitor such sporadic events, while preserving the performance and energy consumption of the current system.

\section{ACKNOWLEDGMENT}

This research is partially funded by imec and Flanders Innovation \& Entrepreneurship (VLAIO) in the context of the WiPeR project, and by the Research Fund KU Leuven.

\section{REFERENCES}

[1] A. Sabato et al., "Wireless mems-based accelerometer sensor boards for structural vibration monitoring: A review," IEEE Sensors Journal, vol. 17 , no. $2,2017$. 
[2] G. Kilic et al., "Testing of wind turbine towers using wireless sensor network and accelerometer," Renewable Energy, vol. 75, 2015.

[3] I. Koene et al., "Iot connected device for vibration analysis and measurement," HardwareX, vol. 7, 2020.

[4] T. Stock et al., "Opportunities of sustainable manufacturing in industry 4.0," Procedia CIRP, vol. 40, 2016, 13th Global Conference on Sustainable Manufacturing - Decoupling Growth from Resource Use.

[5] K. Zhou et al., "Industry 4.0: Towards future industrial opportunities and challenges," in 2015 12th International Conference on Fuzzy Systems and Knowledge Discovery (FSKD), 2015.

[6] H. Raisigel et al., "Autonomous wireless sensor node for building climate conditioning application," in 2010 Fourth International Conference on Sensor Technologies and Applications, 2010.

[7] K. Mekki et al., "A comparative study of lpwan technologies for largescale iot deployment," ICT Express, vol. 5, no. 1, 2019.

[8] M. Centenaro et al., "Long-range communications in unlicensed bands: the rising stars in the iot and smart city scenarios," IEEE Wireless Communications, vol. 23, no. 5, 2016.

[9] U. Raza et al., "Low power wide area networks: An overview," IEEE Communications Surveys Tutorials, vol. 19, no. 2, 2017.

[10] "Bluetooth mesh networking," Bluetooth SIG, March 2019.

[11] M. Baert et al., "The bluetooth mesh standard: An overview and experimental evaluation," Sensors, vol. 18, no. 8, 2018.

[12] L. Hou et al., "Novel industrial wireless sensor networks for machine condition monitoring and fault diagnosis," IEEE Transactions on Instrumentation and Measurement, vol. 61, no. 10, 2012.

[13] L. Hou et al., "Machine fault diagnosis using industrial wireless sensor networks and on-sensor wavelet transform," in IECON 2017-43rd Annual Conference of the IEEE Industrial Electronics Society. IEEE, 2017.

[14] T. Chakraborty et al., "Fall-curve: A novel primitive for iot fault detection and isolation," in Proceedings of the 16th ACM Conference on Embedded Networked Sensor Systems, ser. SenSys '18. New York, NY, USA: Association for Computing Machinery, 2018.

[15] S. Marathe et al., "Currentsense: A novel approach for fault and drift detection in environmental iot sensors," in Proceedings of the International Conference on Internet-of-Things Design and Implementation, 2021.

[16] S. A. S. Al Kazzaz et al., "Experimental investigations on induction machine condition monitoring and fault diagnosis using digital signal processing techniques," Electric Power Systems Research, vol. 65, no. 3, 2003.

[17] J. Shiroishi et al., "Bearing condition diagnostics via vibration and acoustic emission measurements," Mechanical systems and signal processing, vol. 11, no. 5, 1997.

[18] A. Gaglione et al., "Energy neutral operation of vibration energyharvesting sensor networks for bridge applications," in Proceedings of the 2018 International Conference on Embedded Wireless Systems and Networks, ser. EWSN'18, 2018.

[19] G. Feltrin et al., "Vibration monitoring of a footbridge with a wireless sensor network," Journal of Vibration and Control, vol. 19, no. 15, 2013.

[20] J. P. Lynch et al., "Issues in wireless structural damage monitoring technologies," in Proc. of the Third World Conference on Structural Control, vol. 2, 2002.

[21] S. Kim et al., "Health monitoring of civil infrastructures using wireless sensor networks," in Proceedings of the 6th International Conference on Information Processing in Sensor Networks, ser. IPSN '07. New York, NY, USA: Association for Computing Machinery, 2007.

[22] F. Adelantado et al., "Understanding the limits of lorawan," IEEE Communications Magazine, vol. 55, no. 9, 2017.

[23] D. Jung et al., "Vibration analysis for iot enabled predictive maintenance," in 2017 IEEE 33rd International Conference on Data Engineering (ICDE), 2017, pp. 1271-1282.

[24] M. Afanasov et al., "Battery-less zero-maintenance embedded sensing at the mithræum of circus maximus," in Proceedings of the 18th Conference on Embedded Networked Sensor Systems, ser. SenSys '20, 2020.

[25] V. J. Hodge et al., "Wireless sensor networks for condition monitoring in the railway industry: A survey," IEEE Transactions on Intelligent Transportation Systems, vol. 16, no. 3, 2015.

[26] A. S. Thangarajan et al., "Deterministic 40 year battery lifetime through a hybrid perpetual sensing platform (hyper)," in Proceedings of The 10th International Conference on the Internet of Things (IoT 2020). Association for Computing Machinery, 2020.
[27] F. Yang et al., " $\mu$ pnp: Plug and play peripherals for the internet of things," in Proceedings of the Tenth European Conference on Computer Systems, ser. EuroSys '15. Association for Computing Machinery, 2015.

[28] J. Polastre et al., "Telos: enabling ultra-low power wireless research," in IPSN 2005. Fourth International Symposium on Information Processing in Sensor Networks, 2005., 2005.

[29] N. Jackson et al., "Capacity over capacitance for reliable energy harvesting sensors," in Proceedings of the 18th International Conference on Information Processing in Sensor Networks, ser. IPSN '19. New York, NY, USA: Association for Computing Machinery, 2019.

[30] "Nordic semiconductor nRF52832 product specification v1.4," Nordic Semiconductor, October 2017.

[31] Y.-H. Kim et al., "Condition monitoring of low speed bearings: A comparative study of the ultrasound technique versus vibration measurements," in Engineering Asset Management, J. Mathew et al., Eds. London: Springer London, 2006.

[32] P. J. Tavner, "Review of condition monitoring of rotating electrical machines," IET Electric Power Applications, vol. 2, no. 4, 2008.

[33] R. B. Randall et al., "Rolling element bearing diagnostics-a tutorial," Mechanical Systems and Signal Processing, vol. 25, no. 2, 2011.

[34] "LSM6DS3: inemo inertial module always-on 3d accelerometer and 3d gyroscope," 2016.

[35] G. S. Ramachandran et al., " $\mu$ pnp-wan: Experiences with lora and its deployment in dr congo," in 2017 9th International Conference on Communication Systems and Networks (COMSNETS), 2017.

[36] C. Gomez et al., "A sigfox energy consumption model," Sensors, vol. 19, no. 3, 2019

[37] "EVE ER14505, technical specification."

[38] C. W. R. U. Bearing Data Center. CWRU vibration dataset with faulty bearings

[39] W. A. Smith et al., "Rolling element bearing diagnostics using the case western reserve university data: A benchmark study," Mechanical Systems and Signal Processing, vol. 64-65, 2015.

[40] E. Y. Kim et al., "Condition monitoring of low speed bearings: A comparative study of the ultrasound technique versus vibration measurements," Australian Journal of Mechanical Engineering, vol. 5, no. 2, 2008.

[41] M. Entezami et al., "Perspectives on railway axle bearing condition monitoring," Proceedings of the Institution of Mechanical Engineers, Part F: Journal of Rail and Rapid Transit, vol. 234, no. 1, 2020.

[42] B. P. Graney et al., "Rolling element bearing analysis," Materials Evaluation, vol. 70, no. 1, 2012.

[43] S. Kim et al., "Flush: A reliable bulk transport protocol for multihop wireless networks," in Proceedings of the 5th International Conference on Embedded Networked Sensor Systems, ser. SenSys '07. New York, NY, USA: Association for Computing Machinery, 2007.

[44] J. Medina-García et al., "A wireless sensor system for real-time monitoring and fault detection of motor arrays," Sensors, vol. 17, no. 3, 2017.

[45] D. Catenazzo et al. "On the use of wireless sensor networks in preventative maintenance for industry 4.0," in 2018 12th International Conference on Sensing Technology (ICST). IEEE, 2018.

[46] V. M. D. Jagannath et al., "Wibeam:wireless bearing monitoring system," in 2007 2nd International Conference on Communication Systems Software and Middleware, 2007.

[47] E. Cabal-Yepez et al., "Reconfigurable monitoring system for timefrequency analysis on industrial equipment through stft and dwt," IEEE Transactions on Industrial Informatics, vol. 9, no. 2, 2013.

[48] K. Shahzad et al., "Condition monitoring in industry 4.0-design challenges and possibilities: A case study," in 2018 Workshop on Metrology for Industry 4.0 and IoT, 2018.

[49] T. Nyländen et al., "Low-power reconfigurable miniature sensor nodes for condition monitoring," International Journal of Parallel Programming, vol. 43, no. 1, 2015.

[50] N. Tandon et al., "A review of vibration and acoustic measurement methods for the detection of defects in rolling element bearings," Tribology International, vol. 32, no. 8, 1999.

[51] Prabal Dutta et al., "Design of a wireless sensor network platform for detecting rare, random, and ephemeral events," in IPSN 2005. Fourth International Symposium on Information Processing in Sensor Networks, 2005., 2005. 